\title{
The role of peripheral brain-derived neurotrophic factor in chronic osteoarthritic joint pain
}

\author{
Vwaire Orhurhu ${ }^{1}$, Robert $\mathrm{Chu}^{2}$, Sebele Ogunsola ${ }^{3}$, Loretta Akpala ${ }^{4}$, Mariam Salisu Orhurhu ${ }^{1}$, Ivan Urits ${ }^{5}$, \\ Anh L. Ngo ${ }^{6,7}$, Omar Viswanath ${ }^{8,9,10}$, Alan D. Kaye ${ }^{11}$
}

${ }^{1}$ Department of Anesthesia, Critical Care, and Pain Medicine, Massachusetts General Hospital, Harvard Medical School, Boston, MA, USA; ${ }^{2}$ Johns Hopkins School of Medicine, Baltimore, MD, USA; ${ }^{3}$ Department of Anatomy, Case Western Reserve University School of Medicine, Cleveland, OH, USA; ${ }^{4}$ Department of Emergency Medicine, Massachusetts General Hospital, Harvard Medical School, Boston, MA, USA; ${ }^{5}$ Department of Anesthesiology, Critical Care, and Pain Medicine, Beth Israel Deaconess Medical Center, Harvard Medical School, Boston, MA, USA; ${ }^{6}$ Harvard Medical School, Boston, MA, USA; ${ }^{7}$ Department of Pain Medicine, Pain Specialty Group, Newington, NH, USA; ${ }^{8}$ Department of Anesthesiology, Creighton University School of Medicine, Omaha, NE, USA; 'Valley Anesthesiology and Pain Consultants-Envision Physician Services, Phoenix, AZ, USA; ${ }^{10}$ Department of Anesthesiology, University of Arizona College of Medicine-Phoenix, Phoenix, AZ, USA; ${ }^{11}$ Departments of Anesthesiology and Pharmacology, Toxicology, and Neurosciences, Louisiana State University School of Medicine, Shreveport, LA, USA

Correspondence to: Vwaire Orhurhu, MD, MPH. Department of Anesthesia, Critical Care, and Pain Medicine, Massachusetts General Hospital, Harvard Medical School, Boston, MA, USA. Email: vwo569@mail.harvard.edu.

Provenance and Peer Review: This article was commissioned by the editorial office, Annals of Palliative Medicine. The article did not undergo external peer review.

Comment on: Gowler PRW, Li L, Woodhams SG, et al. Peripheral brain-derived neurotrophic factor contributes to chronic osteoarthritis joint pain. Pain 2020;161:61-73.

Submitted Apr 05, 2020. Accepted for publication May 18, 2020.

doi: 10.21037/apm-20-888

View this article at: http://dx.doi.org/10.21037/apm-20-888

Brain-derived neurotrophic factor (BDNF) is a critical signaling protein in the human nervous system. It is found in high levels throughout the mammalian brain and spinal cord, as well as in the peripheral circulation (1-3). The multifaceted role of BDNF depends on the developmental stage of the nervous system. Previous research has demonstrated its role in initial development of the nervous system, synaptogenesis, and maintenance of existing neurons (4). Perhaps not surprisingly, therefore, BDNF has also been implicated in several studies to play a role in pathways related to pain perception. In their published work in this issue entitled "Peripheral brain-derived neurotrophic factor contributes to chronic osteoarthritis joint pain," Gowler and colleagues highlight a heretofore uninvestigated role for BDNF in the modulation of pain related to osteoarthritis (OA) (5). This important study provides a potential mechanism behind OA pain and carries promise for future work into disease-modifying therapies to treat OA.

\section{BDNF expression}

\section{Location of expression}

BDNF's expression in many different human tissues contributes to its central role in nervous system regulation. Hofer and colleagues found during initial research that BDNF is expressed in all major regions of the human brain. However, the hippocampus and cerebral cortex were found to be the locations of highest expression of BDNF mRNA (1). BDNF mRNA was further sublocalized to the neocortex, piriform cortex, amygdala, hippocampus, claustrum, thalamus, hypothalamus, and brainstem (2). BDNF immunoreactivity was observed without mRNA expression in several brain regions, as well as the dorsal horn of the spinal cord. Microglia were also found to contain significant repositories of BDNF (6). Subsequent investigations found measurable levels of BDNF in circulating blood serum (3). 


\section{Method of expression}

BDNF's multiplicity of roles in the nervous system may be in part facilitated by several differing protein products created during its synthesis. Similar to other neurotrophins, BDNF is first synthesized in the endoplasmic reticulum as a precursor molecule, pre-proBDNF. After cleavage of the signal sequence, proBDNF, a $\sim 32-\mathrm{kDa}$ polypeptide is formed. Pro-BDNF is then cleaved by proteases into a 13-kDa mature BDNF (mBDNF) (7). Both proBDNF and $\mathrm{mBDNF}$ isoforms are subsequently released as signaling molecules from the cell $(8,9)$.

\section{Function of BDNF}

As with the other neurotrophins, the different isoforms of BDNF bind preferentially to separate cellular receptors. mBDNF binds to the TrkB receptor, causing dimerization, autophosphorylation, and subsequent activation of the MAPK, PI3 kinase, and PLC- $\gamma$ signaling pathways (10). The overall effect of this signal is a pro-survival effect and long-term potentiation in exposed neuronal populations. Conversely, proBDNF exerts its effects primarily through the p75 neurotrophin receptor (11). Interaction between proBDNF, $\mathrm{p} 75$, and a coreceptor named sortilin triggers several intracellular signaling cascades, including RhoA, which is responsible for neuronal growth cone and motility and $\mathrm{NF}-\kappa \mathrm{B}$, which is responsible for neuronal survival during development. However, the most notable action of proBDNF occurs through the JNK pathway, which ultimately leads to cellular apoptosis. Thus, proBDNF plays an important role in long-term depression, as well as neuronal development and remodeling $(4,11)$.

The opposing effects of mBDNF and proBDNF have been described as a "yin-yang" system that controls synaptic plasticity (12). Indeed, the ratio of mBDNF to proBDNF has been shown to increase between the postnatal period and adulthood (13). Decreased levels of BDNF have been observed in Alzheimer's disease, and normal aging $(14,15)$, while derangements in the ratio of mBDNF to proBDNF have also been observed in Alzheimer's disease and other neurodegenerative pathologies (14).

\section{BDNF and pain}

Perhaps unsurprisingly given its vital role throughout the nervous system, BDNF has also been implicated in chronic pain pathologies related to its ability to drive sensitization of pain pathways. BDNF signaling has been shown to play a role in inflammatory pain via actions in sensory neurons. In this regard, BDNF-knockout mice were demonstrated to display attenuated inflammatory pain responses (16). In the period immediately following peripheral nerve injury, BDNF has been shown to mediate the transition between acute and chronic pain. Experiments where BDNF activity was attenuated via genetic knockdown demonstrated decreased development of allodynia and hyperalgesia in the post-injury period in BDNF-deficient subjects (6). This effect of BDNF can be seen in both the peripheral sensory neuron and the spinal cord, where BDNF is released by microglia (6). BDNF action to increase neuronal hyperexcitability and therefore pain perception in the spinal cord can be explained through both its activation of dorsal horn NMDA receptors and its attenuation of inhibitory GABA signaling by blocking action of the KCC2 potassium-chloride channel (17).

Centrally, BDNF has been implicated in chronic pain states through its effects on descending inhibitory pathways. Neurons in the periaqueductal gray have been shown to release BDNF in response to inflammatory insult, increasing sensitization to pain via NMDA receptor signaling (18). Peripheral serum levels of BDNF have been shown to rise in humans over the course of withdrawal from opioid drugs, perhaps indicating central resensitization to pain (19). Higher serum levels of BDNF are also correlated with lower amounts of descending modulation of pain pathways, as well as decreased tolerance to heat pain (20).

\section{OA summary}

$\mathrm{OA}$ is a common degenerative disease of joints. OA of the knee and hip has increased greatly in recent years due to the aging of the population and now represents the $11^{\text {th }}$-leading cause of disability worldwide. One analysis showed that OA costs could total up to $0.25 \%$ to $0.50 \%$ of a country's gross domestic product (GDP) (21).

The role of BDNF in this debilitating disease has been investigated in several studies to date. Comparison of knee OA patients versus healthy controls found that BDNF levels were significantly elevated in the serum of OA patients but were decreased in the synovial fluid compared to healthy controls. Serum BDNF levels were positively correlated with self-reported pain levels in OA patients (22).

Despite BDNF's putative role in the generation of OA joint pain, it has also been shown to correlate to the therapeutic response to exercise in OA patients. Analysis of 
women with knee OA following a 12-week exercise program found increased serum levels of BDNF in tandem with increased functional status and decreased pain perception (23). A similar finding was reported in a randomized trial evaluating the efficacy of whole body vibration (WBV) therapy in women with knee OA. Women treated with WBV demonstrated increased BDNF levels as well as increased muscle strength, and there was a significant correlation between BDNF level and muscle strength (24).

\section{Commentary}

The recent work published by Gowler and colleagues further explores the relationship between BDNF expression and OA pain. The investigation tests this relationship in both human and animal models. Examination of synovium from human OA patients revealed the presence of BDNF and TrkB mRNA, and TrkB mRNA levels were significantly correlated with expression of the proinflammatory protein CX3CL1 (fractalkine). However, the researchers did not find a significant relationship between BDNF and CX3CL1 expression. In addition, there was not a significant difference in BDNF or TrkB expression between samples based on histologic classification as high- versus lowinflammation states.

A similar analysis was conducted in rats treated with monosodium iodoacetate (MIA), a known model for OA joint inflammation and pain. A multilevel model analysis revealed increased levels of TrkB in MIA-injected rats. ELISA indicated increased BDNF expression in these rats as well. In total, these results suggest an increased concentration of BDNF and TrkB in OA joints.

Armed with these results, the researchers subsequently investigated what role BDNF played in OA inflammation. Intraarticular injection of BDNF at 21 days post-MIA injection resulted in increased pain behavior [e.g., paw withdrawal threshold (PWT) or weight-bearing asymmetry] compared with MIA-injected rats treated with saline. Increased pain behavior manifested on average 5 days after BDNF injection and lasted until the end of the study period. Interestingly, the same BDNF injection in rats not treated with MIA did not result in increased pain behavior. BDNF pain sensitization in OA joints, therefore, seems to require an underlying $\mathrm{OA}$ pathology to be present and does not induce pain in healthy joints.

The effect of BDNF sequestration on $\mathrm{OA}$ joint pain was then investigated. A TrkB-Fc chimera selective for BDNF was injected into the affected joint. This injection significantly decreased weight-bearing asymmetry in both chemical and surgical models of OA compared to a control IgG injection. PWTs were not significantly changed compared to controls. The authors then elegantly repeated the experiment in the contralateral knee. Injected TrkB-Fc failed to decrease pain behavior when injected into the contralateral joint from the OA model, thus demonstrating that the sequestration effects of TrkB-Fc are confined to the local environment. A final study was conducted to determine the length of action of the TrkB-Fc treatment. It was determined that no beneficial effects on pain behavior were seen starting at the 24-hour post-injection mark, thus suggesting the period of activity of TrkB-Fc is less than one day.

In this investigation, Gowler and colleagues have convincingly outlined a role for BDNF and its receptor TrkB in the mediation of OA joint pain. OA joints displayed higher levels of BDNF and $\operatorname{TrkB}$, although it remains unclear the precise relationship between levels of these proteins and the degree of histologically observed joint degeneration in human subjects. Nevertheless, the crux of this investigation to the addition of previous literature is the demonstration of a BDNF-induced increase in pain behavior, which is then subsequently reversed by sequestration of BDNF via TrkB-Fc chimera. These results strongly suggest that $\mathrm{BDNF}$ is not elevated in $\mathrm{OA}$ joints merely as a side effect of joint pathology, but instead play a crucial causal role in the generation of pain during OA. BDNF injection was not able to cause a similar increase in pain activity in non-OA rats, implying that BDNF is a necessary but not sufficient component of OA joint pain. More generally, this investigation leaves the exact mechanism of BDNF-mediated joint pain in question. In this regard, further research must be conducted to determine how BDNF leads to increased pain in OA and if any other targetable steps in this pathway exist. However, this investigation represents a definite addition to the core of knowledge surrounding OA and proposes a strong first step towards developing novel therapeutics for OA pain.

The exact details of this posited therapy, sequestration of BDNF or blockade of signaling action, must be further investigated and refined, however. Unfortunately, the authors of this study determined that the action of TrkB$\mathrm{Fc}$ in sequestering BDNF lasts at most 24 hours. Since this therapy is administered intraarticularly and does not seem to achieve systemic effects, daily TrkB-Fc injections seem like an impractical delivery method for this promising therapy. An effective anti-BDNF therapeutic option would have to achieve longer duration of action within the joint, 
allowing for less frequent injections. Additionally, the potential adverse effects of blocking BDNF action must be considered in any therapy. As the authors mention in their discussion, previous therapies aimed at blocking a similar neurotrophin, nerve growth factor (NGF), while effective, also carried significant adverse effects. Tanezumab, an antiNGF monoclonal antibody, was found to increase incidence of rapidly progressive OA (RPOA), especially in cases of high dosage, concomitant non-steroidal anti-inflammatory drug (NSAID) use, and pre-existing subchondral joint insufficiency (25). BDNF-blocking therapies may face a similar conundrum, especially given BDNF's role in maintaining neuronal structures throughout the rest of the body. Nevertheless, these results have suggested an exciting new avenue for potential treatments for OA pain. We eagerly await additional work that builds on Gowler and colleagues' fundamental findings in developing an end product that successfully improves quality of life for OA patients.

\section{Acknowledgments}

Funding: None.

\section{Footnote}

Conflicts of Interest: All authors have completed the ICMJE uniform disclosure form (available at http://dx.doi. org/10.21037/apm-20-888). The authors have no conflicts of interest to declare.

Ethical Statement: The authors are accountable for all aspects of the work in ensuring that questions related to the accuracy or integrity of any part of the work are appropriately investigated and resolved.

Open Access Statement: This is an Open Access article distributed in accordance with the Creative Commons Attribution-NonCommercial-NoDerivs 4.0 International License (CC BY-NC-ND 4.0), which permits the noncommercial replication and distribution of the article with the strict proviso that no changes or edits are made and the original work is properly cited (including links to both the formal publication through the relevant DOI and the license). See: https://creativecommons.org/licenses/by-nc-nd/4.0/.

\section{References}

1. Hofer M, Pagliusi SR, Hohn A, et al. Regional distribution of brain-derived neurotrophic factor mRNA in the adult mouse brain. EMBO J 1990;9:2459-64.

2. Yan Q, Rosenfeld R, Matheson C, et al. Expression of brain-derived neurotrophic factor protein in the adult rat central nervous system. Neuroscience 1997;78:431-48.

3. Palma-Álvarez RF, Ros-Cucurull E, Amaro-Hosey K, et al. Peripheral levels of BDNF and opiate-use disorder: literature review and update. Rev Neurosci 2017;28:499-508.

4. Kowiański P, Lietzau G, Czuba E, et al. BDNF: a key factor with multipotent impact on brain signaling and synaptic plasticity. Cell Mol Neurobiol 2018;38:579-93.

5. Gowler PRW, Li L, Woodhams SG, et al. Peripheral brain-derived neurotrophic factor contributes to chronic osteoarthritis joint pain. Pain 2020;161:61-73.

6. Coull JAM, Beggs S, Boudreau D, et al. BDNF from microglia causes the shift in neuronal anion gradient underlying neuropathic pain. Nature 2005;438:1017-21.

7. Yang B, Yang C, Ren Q, et al. Regional differences in the expression of brain-derived neurotrophic factor (BDNF) pro-peptide, proBDNF and preproBDNF in the brain confer stress resilience. Eur Arch Psychiatry Clin Neurosci 2016;266:765-9.

8. Conner JM, Lauterborn JC, Yan Q, et al. Distribution of brain-derived neurotrophic factor (BDNF) protein and mRNA in the normal adult rat CNS: evidence for anterograde axonal transport. J Neurosci 1997;17:2295-313.

9. Yang F, Je HS, Ji Y, et al. Pro-BDNF-induced synaptic depression and retraction at developing neuromuscular synapses. J Cell Biol 2009;185:727-41.

10. Foltran RB, Diaz SL. BDNF isoforms: a round trip ticket between neurogenesis and serotonin? J Neurochem 2016;138:204-21.

11. Woo NH, Teng HK, Siao CJ, et al. Activation of p75NTR by proBDNF facilitates hippocampal long-term depression. Nat Neurosci 2005;8:1069-77.

12. Lu B, Pang PT, Woo NH. The yin and yang of neurotrophin action. Nat Rev Neurosci 2005;6:603-14.

13. Yang J, Harte-Hargrove LC, Siao CJ, et al. proBDNF negatively regulates neuronal remodeling, synaptic transmission, and synaptic plasticity in hippocampus. Cell Rep 2014;7:796-806.

14. Bharani KL, Ledreux A, Gilmore A, et al. Serum proBDNF levels correlate with phospho-tau staining in Alzheimer's disease. Neurobiol Aging 2020;87:49-59. 
15. Erickson KI, Prakash RS, Voss MW, et al. BrainDerived Neurotrophic Factor Is Associated with AgeRelated Decline in Hippocampal Volume. J Neurosci 2010;30:5368-75.

16. Lalisse S, Hua J, Lenoir M, et al. Sensory neuronal P2RX4 receptors controls BDNF signaling in inflammatory pain. Sci Rep 2018;8:964.

17. Nijs J, Meeus M, Versijpt J, et al. Brain-derived neurotrophic factor as a driving force behind neuroplasticity in neuropathic and central sensitization pain: a new therapeutic target? Expert Opin Ther Targets 2015;19:565-76.

18. Guo W. Supraspinal brain-derived neurotrophic factor signaling: a novel mechanism for descending pain facilitation. J Neurosci 2006;26:126-37.

19. Zhang K, Jiang H, Zhang Q, et al. Brain-derived neurotrophic factor serum levels in heroin-dependent patients after 26 weeks of withdrawal. Compr Psychiatry 2016;65:150-5.

20. Dussán-Sarria JA, da Silva NRJ, Deitos A, et al. Higher cortical facilitation and serum BDNF are associated with increased sensitivity to heat pain and reduced

Cite this article as: Orhurhu V, Chu R, Ogunsola S, Akpala L, Orhurhu MS, Urits I, Ngo AL, Viswanath O, Kaye AD. The role of peripheral brain-derived neurotrophic factor in chronic osteoarthritic joint pain. Ann Palliat Med 2020;9(4):1361-1365. doi: 10.21037/apm-20-888 endogenous pain inhibition in healthy males. Pain Med 2018;19:1578-86.

21. Puig-Junoy J, Ruiz Zamora A. Socio-economic costs of osteoarthritis: A systematic review of cost-of-illness studies. Semin Arthritis Rheum 2015;44:531-41.

22. Simão AP, Mendonça VA, de Oliveira Almeida TM, et al. Involvement of BDNF in knee osteoarthritis: the relationship with inflammation and clinical parameters. Rheumatol Int 2014;34:1153-7.

23. Gomes WF, Lacerda ACR, Mendonça VA, et al. Effect of exercise on the plasma BDNF levels in elderly women with knee osteoarthritis. Rheumatol Int 2014;34:841-6.

24. Simão AP, Mendonça VA, Avelar NCP, et al. Whole Body Vibration Training on Muscle Strength and Brain-Derived Neurotrophic Factor Levels in Elderly Woman With Knee Osteoarthritis: A Randomized Clinical Trial Study. Front Physiol 2019;10:756.

25. Hochberg MC, Tive LA, Abramson SB, et al. When Is osteonecrosis not osteonecrosis?: adjudication of reported serious adverse joint events in the tanezumab clinical development program. Arthritis Rheumatol 2016;68:382-91. 